IP Periodica Polytechnica Civil Engineering

\author{
62(2), pp. 494-507, 2018 \\ https://doi.org/10.3311/PPci.11537 \\ Creative Commons Attribution (i)
}

RESEARCH ARTICLE

\section{Structural Reliability Assessment Based on the Improved Constrained Differential Evolution Algorithm}

\author{
Mohammad Zaeimi $^{1}$, Ali Ghoddosian ${ }^{1 *}$
}

Received 30 September 2017; Revised 19 December 2017; Accepted 08 January 2018

\begin{abstract}
In this work, the reliability analysis is employed to take into account the uncertainties in a structure. Reliability analysis is a tool to compute the probability of failure corresponding to a given failure mode. In this study, one of the most commonly used reliability analysis method namely first order reliability method is used to calculate the probability of failure. Since finding the most probable point (MPP) or design point is a constrained optimization problem, in contrast to all the previous studies based on the penalty function method or the preference of the feasible solutions technique, in this study one of the latest versions of the differential evolution metaheuristic algorithm named improved $(\mu+\lambda)$-constrained differential evolution (ICDE) based on the multi-objective constraint-handling technique is utilized. The ICDE is very easy to implement because there is no need to the time-consuming task of fine tuning of the penalty parameters. Several test problems are used to verify the accuracy and efficiency of the ICDE. The statistical comparisons revealed that the performance of ICDE is better than or comparable with the other considered methods. Also, it shows acceptable convergence rate in the process of finding the design point. According to the results and easier implementation of ICDE, it can be expected that the proposed method would become a robust alternative to the penalty function based methods for the reliability assessment problems in the future works.
\end{abstract}

\section{Keywords}

reliability analysis, FORM, metaheuristic, ICDE algorithm, multi-objective constraint handling

\section{Introduction}

In this study, the reliability analysis is employed to take into account the uncertainties in a structure. Reliability is defined as the probability that an item (e.g. structure or part of a structure) will adequately perform its specified purpose for a specified period of time under specified environmental conditions [1]. Probability theory is the foundation of reliability analysis which is a tool to compute the reliability index or the probability of failure corresponding to a given failure mode (also known as limit state function) or for the entire system [2]. Since the analytical or numerical evaluation of the probability of failure has some difficulties, different categories of approximate methods are introduced [3-6].

Among these, two more commonly used approaches are the moment methods and simulation methods. The First Order Reliability Method (FORM) as a moment method and the Monte-Carlo simulation (MCS) as a sampling method are the most popular for structural reliability analysis. In comparison to the FORM, The application of the MCS is relatively recent because of the need of powerful computers. The FORM has advantages over the MSC for its computational efficiency, especially when the probability of failure is very small [7]. Because of the efficiency, effectiveness and simplicity of FORM, it has been widely used for reliability analysis and reliability based design optimization [8].

One of the most important elements of the FORM solution is the MPP (most probable point which is proposed by Hasofer and Lind $[9,10]$. In order to find this point, one needs to solve a constrained optimization problem whose objective function is the minimum distance from the origin of standard normal space to the limit state function. This minimum distance is called reliability index or Hasofer-Lind's index [7].

Various algorithms with gradient computations have been proposed to solve the above optimization problem [11]. The commonly used algorithms among them are the Hasofer-Lind and Rackwitz-Fiessler (HL-RF) algorithm [9, 10, 12], modified HL-RF (MHL-RF) [11] and Improved HL-RF (iHL-RF) [13]. The main drawbacks of these algorithms are the computation of the gradients with respect to the random variables 
and trapping in the local optimum solutions. Therefore it is essential to use gradient free algorithms which have the ability to find global or near-global optimum solution.

Therefore In the last decade, the metaheuristic algorithms are used in the reliability analysis. Two main characteristics of these algorithms are the exploration and exploitation. In the exploration phase, they explore the search space more efficiently and in the exploitation phase, they search the current best solutions and select the best candidates [14].

For the first time, Elegbede [4] used the particle swarm optimization (PSO) method to determine reliability index. He applied the exterior penalty method to convert the constrained optimization problem to an unconstrained one. The results show that this method can find the design point and the reliability index with a good accuracy. Also, the dependency of computing time on the initial population size and number of iterations was investigated. By combining the benefits of the shredding and learning operators, Wang and Ghosn [15] proposed a new hybrid genetic search algorithm which is suitable for solving structural reliability problems. They used the Shredding operator to reduce the number of structural analyses and obtain the value of the reliability index.

Yan et al. [16] applied the socio-political evolutionary algorithm, the imperialist competitive algorithm (ICA), combined with penalty function method to solve the reliability index optimization problem. The results showed the precision and good feasibility of the proposed model. Zhao et al. [17] employed the chaotic particle swarm optimization (CPSO) method to conduct structural reliability analysis. The CPSO is a combination of the well-known PSO with a chaotic system to improve the global search performance of the PSO. They reported the CPSO is a good approach to find accurate design point and reliability index.

In a similar study, the charged system search (CSS) algorithm is used by Kaveh et al. [5] to solve aforementioned constrained optimization. To apply the CSS in reliability analysis, the penalty method is used. For carrying out a comparative study, Kaveh and Ghazaan [18] utilize four metaheuristic algorithms consisting of the improved ray optimization (IRO), democratic particle swarm optimization (DPSO), colliding bodies optimization (CBO) and enhanced colliding bodies optimization (ECBO) with the penalty function to estimate failure probability of problems. The results showed the suitability, efficiency and good accuracy of these algorithms. Also, they had the same performance. Chakri et al. [6] proposed an improved version of the bat algorithm named improved bat algorithm (iBA) and an adaptive penalty function to obtain the reliability index. After comparison iBA with the other metaheuristic algorithms, they reported that the iBA is more efficient and reliable.

It can be found that all the previous studies which were based on the metaheuristic algorithms, used the penalty function method or the preference of the feasible solutions technique to handle the constraints, while the improved $(\mu+\lambda)$-constrained differential evolution (ICDE) algorithm, which is based on multi-objective constraint-handling technique is used in this paper for the reliability assessment problem for the first time. In section 2 a brief description of the reliability assessment problem is proposed. The ICDE algorithm is then presented briefly in section 3. Three type numerical examples, benchmark test functions and structural examples are presented in section 4. Finally, the conclusion is proposed in Section 5.

\section{Reliability assessment}

By performing a probabilistic reliability analysis, the probability of failure or reliability index corresponding to the limit state function or failure mode can be obtained. The limit state function $g(X)$ is a function of random variables, $X=\left(X_{1}, X_{2}\right.$, ..., $\left.X_{n}\right)$ which separates the failure region $(g(X)<0)$ and safe region $(g(X)>0)$. Also $g(X)$ is called performance function. The probability of failure $P_{f}$ is defined as [3]:

$$
P_{f}=\operatorname{Prob}\{g(\boldsymbol{X}) \leq 0\}=\int_{g(\boldsymbol{X}) \leq 0} f_{x}(\boldsymbol{x}) d \boldsymbol{x}
$$

Where $f x(x)$ is the joint probability density function (PDF) of $X$. Then the reliability $R$ is obtained by:

$$
R=1-P_{f}=\int_{g(\boldsymbol{X})>0} f_{x}(\boldsymbol{x}) d \boldsymbol{x}
$$

Because of some difficulties to solve the above multi-dimensional integral [4], one of the most commonly used reliability analysis technique namely FORM (First Order Reliability Method) has been used in this paper.

It should be noted that the development of FORM can be traced to the first order second moment (FOSM) method which is proposed by Cornell $[19,20]$. He proposed an index related to the probability of failure to estimate the structural reliability. However, it is not easy to use Cornell index for measuring the probability of failure because of the strong dependency on the mathematical formulation of the limit state function (two different equivalent formulations of the same limit state function result in different values of Cornell index). In order to overcome this limitation, an alternative approach based on the concept of the design point was proposed by Hasofer and Lind which is introduced in the following.

First, the random vector $X$ is mapped into an independent standard normal vector $U$ by using the Rosenblatt transformation [21] to simplify the shape of the $f x(x)$. The standard normal variable has a zero mean and unit standard deviation. After the transformation the performance function becomes $g(U)$. Next, the integration boundary $g(U)=0$ is linearized by the first order Taylor expansion at the most probable point (MPP) [1]. The MPP is a point that has minimum distance from the origin in the standard normal space to the performance $g(U)$ $=0$. Therefore, finding the MPP is a constrained optimization problem with an equality constraint as follow: 


$$
\left\{\begin{array}{l}
\beta=\min \boldsymbol{u} \\
\text { subject to }
\end{array} g(\boldsymbol{u})=0\right.
$$

Where $\beta$ is the Hasofer-Lind reliability index or the reliability index $[9,10,12]$. To solve Eq. (3), a usual approach is to transform the equality constraint into the following inequality one:

$$
|g(\boldsymbol{u})|-\delta \leq 0
$$

Where $\delta$ is a tolerance value. So the probability of failure is approximated based on the reliability index as follow:

$$
P_{f}=\Phi(-\beta)
$$

Where $\Phi$ is the standard normal cumulative distribution function (CDF). Note that, in FORM, it is assumed that all random variables have normal distribution. However, some basic variables for most reliability problems are not normally distributed. Therefore, it is necessary to transform the variables with non-normal distributions into the normally distributed ones. The interested reader is referred to literature for more information [3].

\section{Optimization algorithm for reliability assessment}

Since the calculation of the reliability index is a constrained optimization problem, so it is essential to use one of the existing constraint-handling techniques. It can classify these techniques into three main groups [22]: the penalty function methods, the methods based on the preference of the feasible solutions and the multi-objective optimization techniques.

In the penalty function methods, a constrained optimization problem transform into an unconstrained one using the penalty parameters. The main drawback of this method is that it requires a lot of fine-tuning of the penalty parameters to guide the search towards the global optimum. The requirement of this fine tuning should be either minimized or be eliminated by a good constraint-handling technique. When fine tuning is essential, the performance of the algorithm is affected by it [23].

In the methods based on the preference of the feasible solutions, the selection procedure only executes pairwise comparisons. While a feasible solution always has a higher priority over an infeasible one, between two feasible solutions, the one having a better objective function value is given priority; between two infeasible solutions, however, the one having smaller constraint violation is preferred [24]. The main problem of this technique is to maintain diversity in the population to prevent the premature convergence to local optimum [23].

As mentioned earlier, all the previous works based on the metaheuristic algorithms utilized the penalty function method or the preference of the feasible solutions technique to handle the constraints of the reliability assessment problem. In this paper, an improved $(\mu+\lambda)$ constraint differential evolution (ICDE) algorithm is used which is based on the multi-objective constraint-handling technique. The main idea of this technique is to redefine the single objective optimization as a multi-objective optimization in which the objective functions consist of the original objective function and the constraints. In the ICDE algorithm, the population diversity is controlled successfully and also there is no need to the trial and error process of finding appropriate penalty parameters.

The ICDE is the latest versions of differential evolution (DE) algorithm in order to solve constrained optimization problem $[22,25,26]$. It includes two main parts, the Improved $(\mu+\lambda)$-differential evolution (IDE) to search in the design space and the archiving-based adaptive tradeoff model (ArATM) to handle the constraints of the problem. The flowchart of the ICDE is illustrated in Fig. 1. In the following sections, the DE and main parts of the ICDE are proposed briefly. For more details, the interested reader is referred to the work by Jia et al [22].

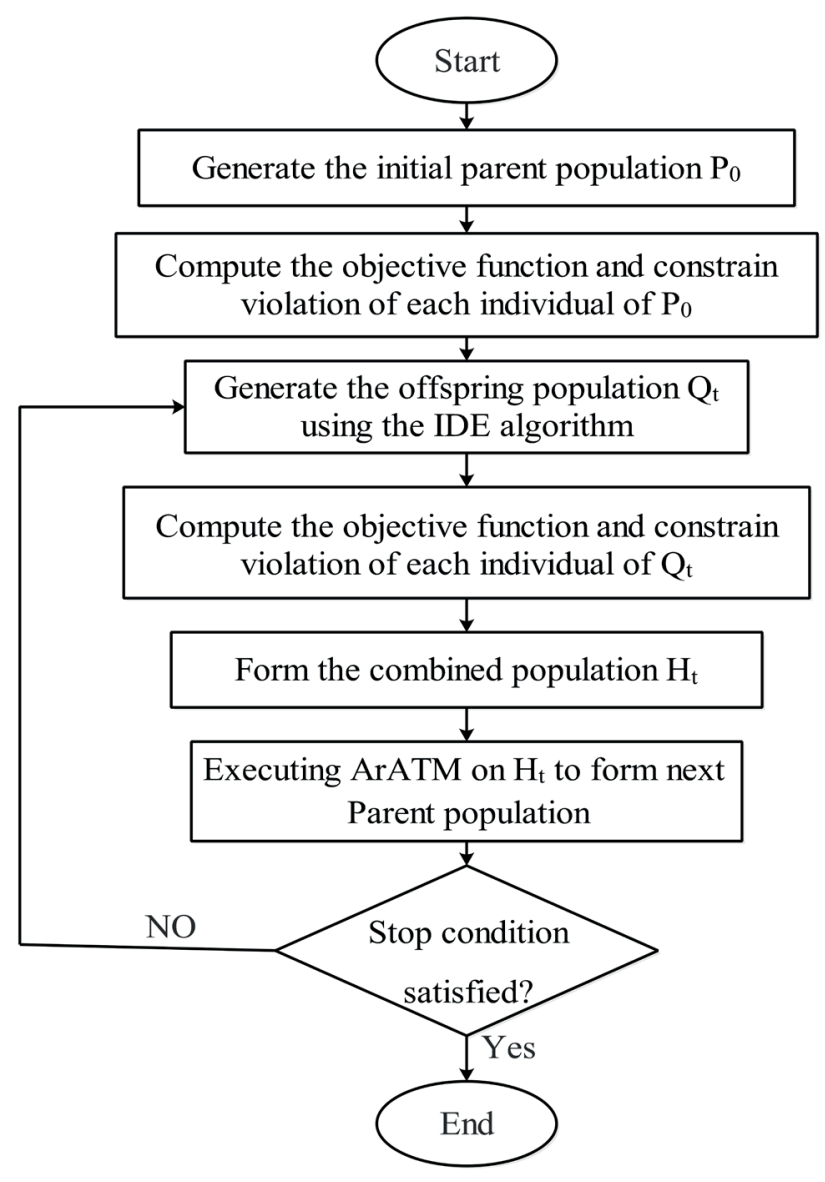

Fig. 1 Flowchart of the ICDE algorithm

\subsection{Differential evolution (DE) algorithm}

The differential evolution (DE) is a population-based algorithm which is developed by Storn and Price [27]. It is one of the most successful evolutionary algorithms (EAs) for the global numerical optimization. In order to evolve the population to the global optimum solution, it utilizes the mutation, crossover, and selection operators. The mutation and crossover are used to produce the population of trial vectors in order to ensure diversity of the population. The procedure of the $\mathrm{DE}$ consists of five main steps as follow: 
Step 1: forming an initial population

After defining both upper and lower bounds of the variables, the initial population $P_{0}$ of $\mu$ individuals (parents) can be generated randomly in the search space as follows:

$$
\boldsymbol{x}_{i}^{0}=\boldsymbol{x}^{l}+\operatorname{rand} .\left(\boldsymbol{x}^{u}-\boldsymbol{x}^{l}\right), i=1,2, \ldots, \mu
$$

Where $\boldsymbol{x}_{i}^{0}$ is the current individual in the initial population ( $t=0$ where $t$ is the generation number) including, $\boldsymbol{x}^{l}$ and $\boldsymbol{x}^{u}$ are the lower bound and upper bound of variables respectively and rand is a uniformly distributed random number between 0 and 1.

Step 2: generating the mutant vectors

In the ICDE four mutation strategies have been employed to generate a mutant vector $\boldsymbol{v}_{i}$ from each current individual as follows [22]:

"rand/1" mutation:

$$
\boldsymbol{v}_{i}^{t}=\boldsymbol{x}_{r_{1}}^{t}+F \cdot\left(\boldsymbol{x}_{r_{2}}^{t}-\boldsymbol{x}_{r_{3}}^{t}\right)
$$

"rand/2" mutation:

$$
\boldsymbol{v}_{i}^{t}=\boldsymbol{x}_{r_{1}}^{t}+F \cdot\left(\boldsymbol{x}_{r_{2}}^{t}-\boldsymbol{x}_{r_{3}}^{t}\right)+F \cdot\left(\boldsymbol{x}_{r_{4}}^{t}-\boldsymbol{x}_{r_{5}}^{t}\right)
$$

"current-to-Rand/1" mutation:

$$
\boldsymbol{v}_{i}^{t}=\boldsymbol{x}_{i}^{t}+F \cdot\left(\boldsymbol{x}_{r_{1}}^{t}-\boldsymbol{x}_{i}^{t}\right)+F \cdot\left(\boldsymbol{x}_{r_{2}}^{t}-\boldsymbol{x}_{r_{3}}^{t}\right)
$$

"current-to-best/1" mutation:

$$
\boldsymbol{v}_{i}^{t}=\boldsymbol{x}_{i}^{t}+F \cdot\left(\boldsymbol{x}_{\text {best }}^{t}-\boldsymbol{x}_{i}^{t}\right)+F \cdot\left(\boldsymbol{x}_{r_{1}}^{t}-\boldsymbol{x}_{r_{2}}^{t}\right)
$$

Where $r_{1}, r_{2}, r_{3}, r_{4}$, and $r_{5}$, are different integers, randomly selected from the set $\{1,2, \ldots, \mu\}$ with the condition of $\left\{r_{1} \neq r_{2}\right.$ $\left.\neq r_{3} \neq r_{4} \neq r_{5}\right\}, \boldsymbol{x}_{\text {best }}^{t}$ and $\boldsymbol{x}_{i}^{t}$ are respectively the best individual and the current individual in the current population (generation number is equal to $t$ ) and $F$ is a randomly selected number between 0 and 1 .

Step 3: checking the boundary constraints

To handle the boundary constraints violation, the component of mutant vector $\boldsymbol{v}_{i}$ is modified as follows [28]:

$$
v_{i, j}^{t}=\left\{\begin{array}{cl}
2 x_{j}^{l}-v_{i, j}^{t} & \text { if } v_{i, j}^{t}<x_{j}^{l} \\
2 x_{j}^{u}-v_{i, j}^{t} & \text { if } v_{i, j}^{t}>x_{j}^{u} \\
v_{i, j}^{t} & \text { otherwise }
\end{array}\right.
$$

As can be seen from the above formulation, if the $j$ th component $v_{i, j}^{t}$ of the mutant vector $\boldsymbol{x}_{i}^{t}$ violates the boundary constraint, $v_{i, j}^{t}$ is reflected back from the violated boundary constraint.

Step 4: using crossover operator to generate trial vectors

After modifying the violated mutant vector, the binomial crossover is applied to produce the trial vector $\boldsymbol{u}_{i}$ by changing some components of the mutant vector as follows:

$$
u_{i, j}^{t}=\left\{\begin{array}{cc}
v_{i, j}^{t} & \text { if rand } \leq C R \text { or } j=j_{\text {rand }} \\
x_{i, j}^{t} & \text { otherwise }
\end{array}\right.
$$

Step 5: comparing the trial vector and current vector

In this step, the trial vector compare with the current vector based on their objective function values and the better one will survive in the next generation:

$$
\boldsymbol{x}_{i}^{t+1}=\left\{\begin{array}{cc}
\boldsymbol{u}_{i}^{t} & \text { if } f\left(\boldsymbol{u}_{i}^{t}\right) \leq f\left(\boldsymbol{x}_{i}^{t}\right) \\
\boldsymbol{x}_{i}^{t} & \text { otherwise }
\end{array}\right.
$$

\subsection{Improved $(\mu+\lambda)$-differential evolution (IDE) algorithm}

The IDE is an improved version of the DE with a better diversity of the population. It is utilized in the ICDE to investigate the search space. In the IDE, the offspring population $Q_{t}$ is produced from the current population $P^{t}$ with $\mu$ individuals. It has three main steps as follows:

Step 1: set $Q_{t}=\varnothing$;

Step 2: generate three offspring for each individual in the current populationas follows:

First offspring $\boldsymbol{y}_{1}$ : executing the "rand/1" mutation strategy and the binomial crossover;

Second offspring $\boldsymbol{y}_{2}$ : executing the "rand/2" mutation strategy and the binomial crossover;

Third offspring $\boldsymbol{y}_{3}$ : executing a new mutation strategy named "current-to-best/1" and the iBGA (improved breeder genetic algorithm) [22];

Step 3: update the offspring population, $Q_{t}=Q_{t} \cup \boldsymbol{y}_{1} \cup \boldsymbol{y}_{2}$ $\cup \boldsymbol{y}_{3}$;

In step 2, the "current-to-rand/best/1" strategy has two phases with different mutation strategy. In the first phase, the "current-to-rand/1" strategy is used to enhance the global search of the population. When the current generation number is more than a predefined threshold generation number, the second phase begins. In the second phase to increase the convergence rate of the population toward the global optimum, the "current-to-best/1" is implemented. The aim of using this strategy is to achieve a good balancing between the diversity and the convergence of the population. In step 3, it can be seen that by performing the above procedure, the offspring population has $\lambda=3 \mu$ individuals.

\subsection{Archiving-based adaptive trade-off model (ArATM)}

There are three situations after combining the offspring population and the parent population in a combined population $H_{t}=P_{t}+Q_{t}$. The situations are the infeasible, semi-feasible and feasible situations. For each of these situations a different constraint-handling mechanism is designed in the ArATM.

In the infeasible situation, all individuals violate the constraints of the problem. The aim of the corresponding mechanism is to guide the infeasible population toward the feasible region in the early stage of the evolution and to maintain its diversity. In this situation, the original problem transformed 
to bi-objective optimization problem and a good tradeoff between two objects, the objective function and the degree of constraint violation, is established. In addition, the individuals those which are not suitable to survive into the next generation will be stored to compete with the individuals of the next combined population $H_{t+1}$. In this way the diversity of the population may be enhanced during the evolutionary process.

In the semi-feasible situation, both feasible and infeasible individuals exist in the combined population $H_{t}$. In this situation, some infeasible individuals which contain important information is also utilized to guide the search toward global optimum. By implementing an adaptive fitness transformation scheme, not only some feasible individuals with small fitness values but also some infeasible individuals with both small degree of constraint violation and small fitness values survive into the next generation.

Finally in the feasible situation, all individuals in the combined population $H_{t}$ are in the feasible region. The comparison between all individuals is implemented only by considering their fitness values. Therefore, $\mu$ individuals with the smallest fitness value constitute the next population $P_{t+1}$. In the next sections, several test problems are employed to investigate the performance of the ICDE in the reliability problems.

\section{Numerical results}

In this section, the ICDE is applied to the different benchmark test problems drawn from the literature. In order to investigate the accuracy and efficiency of the proposed algorithm, each problem is independently solved 20 times. Then the results are compared with the available solutions obtained from the other metaheuristic algorithms based on the penalty function method or the preference of the feasible solutions technique, the HL-RF and sampling methods (i.e. important sampling and Monte-Carlo simulation methods) in the previous studies. It is noted that for all test problems, the common parameters in ICDE are set as follows: $\mu=20, C R=0.8, F=$ 0.9 and $\delta=0.0001$. Note that, when there is no improvement of the solutions after 10 iterations, the process of the optimization will be stopped.

\subsection{Example 1: limit state function with normal basic variables}

Eleven non-linear limit state functions with different number of random variables chosen from references are summarized in Table 1. They can be classified into two groups based on the description of their random variables. The first eight limit state functions have the independent standard normal random variables and the others have the independent normal random variables.

Table 2 presents the optimum reliability index and the corresponding probability of failure obtained by different optimization methods. It can be observed that all metaheuristic methods converge to the same results but better than the ARBIS method (adaptive radial-based importance sampling) which is more efficient than the Monet-Carlo simulation [29]. In Table 3, the optimum design points are proposed. Also, the statistical comparison between the results of ICDE and those are available in the previous studies are presented in table 4 and Fig. 2.

Table 1 Limit state functions and random variables distribution for example 1

\begin{tabular}{|c|c|}
\hline Limit state function & Random variables \\
\hline$g_{1}(x)=5-0.5\left(x_{1}-0.1\right) 2-x_{2}$ & $x_{i}: N(0,1), i=1,2$ \\
\hline$g_{2}(x)=3-x_{2}+\left(4 x_{1}\right)^{2}$ & $x_{i}: N(0,1), i=1,2$ \\
\hline$g_{3}(x)=2-x_{2}-0.1 x_{1}^{2}+0.06 x_{1}$ & $x_{i}: N(0,1), i=1,2$ \\
\hline$g_{4}(x)=0.1\left(x_{1}-x_{2}\right)^{2}-\frac{\left(x_{1}+x_{2}\right)}{\sqrt{2}}+2.5$ & $x_{i}: N(0,1), i=1,2$ \\
\hline$g_{5}(x)=-0.5\left(x_{1}-x_{2}\right)^{2}-\frac{\left(x_{1}+x_{2}\right)}{\sqrt{2}}+3$ & $x_{i}: N(0,1), i=1,2$ \\
\hline $\begin{array}{l}g_{6}(x)=\exp \left(0.4\left(x_{1}+2\right)+6.2\right)- \\
\exp \left(0.3 x_{2}+5\right)-200\end{array}$ & $x_{i}: N(0,1), i=1,2$ \\
\hline$g_{7}(x)=\exp \left(0.2 x_{1}+1.4\right)-x_{2}$ & $x_{i}: N(0,1), i=1,2$ \\
\hline $\boldsymbol{g}_{8}(x)=2+0.015 \sum_{i=1}^{9} x_{i}^{2}-x_{10}$ & $x_{i}: N(0,1), i=1, \ldots, 10$ \\
\hline $\boldsymbol{g}_{9}(x)=x_{1}-\frac{x_{2}}{x_{3}}$ & $\begin{array}{c}x_{1}: N(600,30) \\
x_{2}: N(1000,33) \\
x_{3}: N N(20.1)\end{array}$ \\
\hline$g_{10}(x)=x_{1} x_{2}-146.14$ & $\begin{array}{l}x_{1}: N(78064,11709.9) \\
x_{2}: N(0.0104,0.00156)\end{array}$ \\
\hline $\begin{array}{l}g_{11}(x)=2.5-0.2375\left(x_{1}-x_{2}\right)+ \\
0.00463\left(x_{1}+x_{2}-20\right)^{4}\end{array}$ & $x_{i}: N(10,3), i=1,2$ \\
\hline
\end{tabular}

In Fig. 2, corresponding to each Statistical terms, $N$ indicates the total number of superior performances of an algorithm comparing to the others in the previously mentioned limit state functions. Statistical terms are proposed along the horizontal axis. According to Table 4, there can be three following situations for every statistical term to calculate the value of $N$ in Fig. 2:

1) There is only one algorithm with better results than the others;

2) There are some algorithms that show the identical results while better than the rest;

3) All algorithms show the identical results;

In the above situations, the value of $N$ increased for algorithms with better performances. This process is repeated for all statistical terms and the final results are shown in Fig. 2. From the statistical comparisons in Fig. 2, the performance of the ICDE in terms of the best $\beta$, worst $\beta, \operatorname{avg} \beta, \operatorname{std} \beta$ and std iteration is superior to the other algorithms namely, $\mathrm{CBO}$, DPSO, IRO and ECBO for the eleven test problems which are described in table 1. 
Table 2 Optimum reliability index and corresponding probability of failure obtained by different methods for example 1

\begin{tabular}{|c|c|c|c|c|c|c|c|c|c|c|}
\hline & & ICDE [18] & IRO [18] & DPSO [18] & CBO [18] & ECBO [18] & CSS [5] & PSO [4] & $\mathrm{CPSO}[17]$ & ARBIS [29] \\
\hline \multirow{2}{*}{$g_{1}$} & $\beta$ & 2.9057 & 2.9058 & 2.9056 & 2.9057 & 2.9056 & 2.9060 & 2.9056 & - & - \\
\hline & $P_{f}$ & 0.0018 & 0.0018 & 0.0018 & 0.0018 & 0.0018 & 0.0018 & 0.0018 & - & - \\
\hline \multirow{2}{*}{$g_{2}$} & $\beta$ & 2.9999 & 2.9999 & 2.9999 & 2.9999 & 2.9999 & 3.0000 & - & - & 2.9250 \\
\hline & $P_{f}$ & 0.0014 & 0.0014 & 0.0014 & 0.0014 & 0.0014 & 0.0014 & - & - & 0.0002 \\
\hline \multirow[b]{2}{*}{$g_{3}$} & $\beta$ & 1.9999 & 1.9999 & 1.9999 & 1.9999 & 1.9999 & 2.0000 & - & - & 1.9960 \\
\hline & $P_{f}$ & 0.0228 & 0.0228 & 0.0228 & 0.0228 & 0.0228 & 0.0228 & - & - & 0.0347 \\
\hline \multirow{2}{*}{$g_{4}$} & $\beta$ & 2.4999 & 2.4999 & 2.4999 & 2.4999 & 2.4999 & - & - & - & 2.4180 \\
\hline & $P_{f}$ & 0.0062 & 0.0062 & 0.0062 & 0.0062 & 0.0062 & - & - & - & 0.0042 \\
\hline \multirow{2}{*}{$\boldsymbol{g}_{5}$} & $P_{f}$ & 1.6583 & 1.6582 & 1.6582 & 1.6582 & 1.6583 & - & - & - & 1.6250 \\
\hline & $\mathrm{Pf}$ & 0.0486 & 0.0486 & 0.0486 & 0.0486 & 0.0486 & - & - & - & 0.1050 \\
\hline \multirow[b]{2}{*}{$g_{6}$} & $\beta$ & 2.7099 & 2.7129 & 2.7103 & 2.7109 & 2.7149 & 2.7100 & 2.7099 & 2.7099 & - \\
\hline & $P_{f}$ & 0.0034 & 0.0033 & 0.0034 & 0.0034 & 0.0033 & 0.0034 & 0.0034 & 0.0034 & - \\
\hline \multirow{2}{*}{$g_{7}$} & $\beta$ & 3.3496 & 3.3496 & 3.3496 & 3.3496 & 3.3496 & 3.3500 & 3.4971 & - & - \\
\hline & $P_{f}$ & 0.0004 & 0.0004 & 0.0004 & 0.0004 & 0.0004 & 0.0004 & 0.0004 & - & - \\
\hline \multirow[b]{2}{*}{$g_{8}$} & $\beta$ & 1.9999 & 2.0053 & 2.0000 & 1.9999 & 2.0000 & 2.0000 & - & - & 2.1030 \\
\hline & $P_{f}$ & 0.0228 & 0.0225 & 0.0228 & 0.0228 & 0.0228 & 0.0228 & - & - & 0.0053 \\
\hline \multirow{2}{*}{$g_{9}$} & $\beta$ & 2.2697 & 2.2769 & 2.3413 & 2.2701 & 2.3814 & 2.2696 & 2.2697 & 2.2784 & - \\
\hline & $P_{f}$ & 0.0116 & 0.0114 & 0.0096 & 0.0116 & 0.0086 & 0.0116 & 0.0116 & 0.0114 & - \\
\hline \multirow{2}{*}{$\boldsymbol{g}_{10}$} & $\beta$ & 5.3333 & 5.3370 & 5.3516 & 5.3442 & 5.3387 & 5.3332 & - & - & 5.4430 \\
\hline & $P_{f}$ & $4.82 \mathrm{e}-8$ & $4.72 \mathrm{e}-8$ & $4.35 \mathrm{e}-8$ & $4.35 \mathrm{e}-8$ & $4.67 \mathrm{e}-8$ & $4.82 \mathrm{e}-8$ & - & - & $0.1 .46 \mathrm{e}-07$ \\
\hline \multirow{2}{*}{$g_{11}$} & $\beta$ & 2.4999 & 2.4999 & 2.4999 & 2.4999 & 2.4999 & - & - & 2.5001 & 2.4310 \\
\hline & $P_{f}$ & 0.0062 & 0.0062 & 0.0062 & 0.0062 & 0.0062 & - & - & 0.0062 & 0.0029 \\
\hline
\end{tabular}

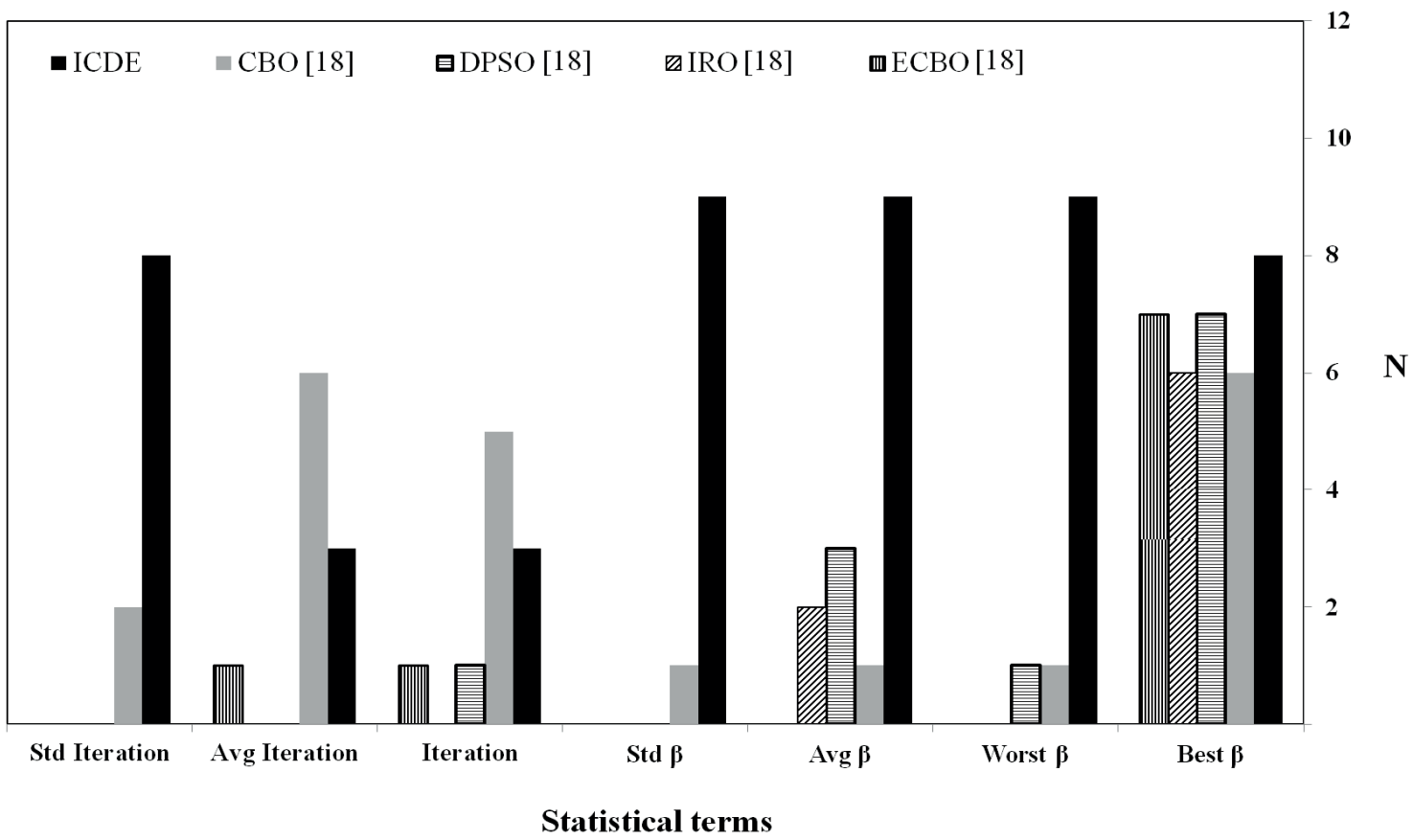

Fig. 2 Comparison of the statistical results for example 1 
Table 3 Optimum design points obtained by different methods for example 1

\begin{tabular}{|c|c|c|c|c|c|c|c|c|c|c|}
\hline & & ICDE [18] & IRO [18] & DPSO [18] & $\mathrm{CBO}[18]$ & ECBO [18] & CSS [5] & PSO [4] & CPSO [17] & ICA [16] \\
\hline \multirow{2}{*}{$g_{1}$} & $x_{1}$ & -2.74079 & -2.75145 & -2.74074 & -2.74499 & -2.74014 & -2.74080 & -2.74163 & - & - \\
\hline & $x_{2}$ & 0.96484 & 0.93450 & 0.96498 & 0.95296 & 0.96677 & 0.96470 & 0.96258 & - & - \\
\hline \multirow{2}{*}{$g_{2}$} & $x_{1}$ & -0.00001 & -0.00325 & -0.00042 & 0.00000 & -0.00625 & 0.00000 & - & - & - \\
\hline & $x_{2}$ & 2.99990 & 2.99990 & 2.99990 & 2.99990 & 2.99991 & 3.00000 & - & - & - \\
\hline \multirow{2}{*}{$g_{3}$} & $x_{1}$ & 0.00001 & -0.00174 & 0.00102 & $-2.35 e-08$ & 0.00132 & $-4.17 e-08$ & - & - & - \\
\hline & $x_{2}$ & 1.99990 & 1.99989 & 1.99980 & 1.99990 & 1.99990 & 2.00000 & - & - & - \\
\hline \multirow{2}{*}{$g_{4}$} & $x_{1}$ & 1.76775 & 1.76760 & 1.76209 & 1.75802 & 1.76847 & - & - & - & - \\
\hline & $x_{2}$ & -1.76778 & 1.76778 & 1.77330 & 1.77742 & 1.76690 & - & - & - & - \\
\hline \multirow{2}{*}{$g_{5}$} & $x_{1}$ & 1.47157 & 1.46990 & 1.46880 & 1.47398 & -0.76116 & - & - & - & - \\
\hline & $x_{2}$ & -0.76445 & -0.76747 & -0.76970 & -0.75980 & 1.47329 & - & - & - & - \\
\hline \multirow{2}{*}{$g_{6}$} & $x_{1}$ & -2.53965 & -2.58720 & -2.52197 & -2.56748 & -2.47706 & -2.53960 & -2.54776 & -2.5407 & - \\
\hline & $x_{2}$ & 0.94537 & 0.81629 & 0.99268 & 0.87017 & 1.11142 & 0.94350 & 0.92355 & 0.94270 & - \\
\hline \multirow{2}{*}{$g_{7}$} & $x_{1}$ & -1.67972 & -1.67975 & -1.68462 & -1.66811 & -1.68013 & -1.67970 & -1.68825 & - & - \\
\hline & $x_{2}$ & 2.89800 & 2.89798 & 2.89521 & 2.90470 & 2.89777 & 2.89810 & 2.89316 & - & - \\
\hline \multirow{10}{*}{$g_{8}$} & $x_{1}$ & -0.00328 & 0.04490 & -0.01055 & 0.00574 & -0.00449 & -0.00018 & - & - & - \\
\hline & $x_{2}$ & 0.00093 & -0.03938 & 0.00725 & 0.00347 & -0.00461 & -0.00012 & - & - & - \\
\hline & $x_{3}$ & 0.00073 & 0.04674 & -0.00950 & 0.00452 & -0.00867 & 0.00011 & - & - & - \\
\hline & $x_{4}$ & 0.00044 & 0.06378 & 0.00037 & -0.00460 & -0.01044 & 0.00099 & - & - & - \\
\hline & $x_{5}$ & -0.00029 & -0.01920 & 0.01194 & -0.00190 & -0.01201 & 0.00266 & - & - & - \\
\hline & $x_{6}$ & 0.00100 & 0.01470 & -0.00670 & -0.01274 & 0.00206 & 0.00023 & - & - & - \\
\hline & $x_{7}$ & 0.00227 & -0.07475 & -0.00161 & -0.00243 & 0.00320 & -0.00082 & - & - & - \\
\hline & $x_{8}$ & 0.00028 & -0.06726 & 0.00509 & 0.00127 & 0.00357 & 0.00005 & - & - & - \\
\hline & $x_{9}$ & 0.00142 & -0.00213 & -0.01465 & 0.00012 & 0.01327 & 0.00345 & - & - & - \\
\hline & $x_{10}$ & 1.99991 & 2.00020 & 1.99991 & 1.99990 & 1.99991 & 2.00000 & - & - & - \\
\hline \multirow{3}{*}{$g_{9}$} & $x_{1}$ & 591.800 & 559.710 & 565.220 & 555.710 & 540.560 & 555.608 & 555.507 & 553.286 & 555.790 \\
\hline & $x_{2}$ & 1003.05 & 1032.300 & 1044.800 & 1030.300 & 1012.900 & 1029.000 & 1028.950 & 1023.074 & 1028.667 \\
\hline & $x_{3}$ & 1.69400 & 1.84440 & 1.84840 & 1.85400 & 1.87380 & 1.85202 & 1.85227 & 1.84909 & 1.85081 \\
\hline \multirow{2}{*}{$g_{10}$} & $x_{1}$ & 59690.2 & 19516.0 & 51622.0 & 20470.0 & 55456.0 & 59684.0 & - & - & - \\
\hline & $x_{2}$ & 0.00244 & 0.00748 & 0.00283 & 0.00713 & 0.00263 & 0.00244 & - & - & - \\
\hline \multirow{2}{*}{$g_{11}$} & $x_{1}$ & 15.3031 & 15.3040 & 15.3140 & 15.3030 & 15.3190 & - & - & 15.3334 & - \\
\hline & $x_{2}$ & 4.69600 & 4.69740 & 4.70800 & 4.69650 & 4.71250 & - & - & 4.72670 & - \\
\hline
\end{tabular}

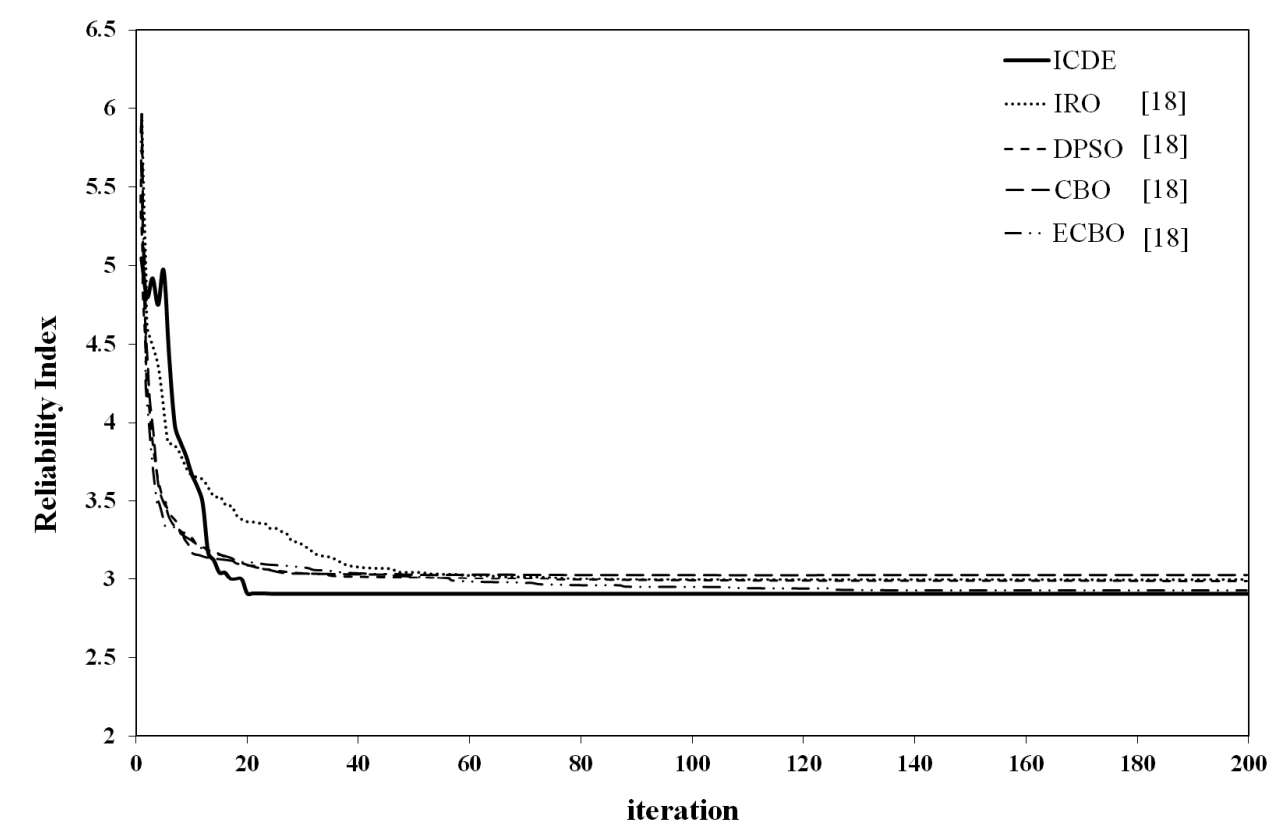

Fig. 3 Comparison of the average values of the reliability index obtained by the different algorithms for g1 in Table 1 
Table 4 Statistical results obtained by ICDE and IRO [18], DPSO [18], CBO [18] and ECBO [18] for example 1

\begin{tabular}{|c|c|c|c|c|c|c|c|c|}
\hline & & Best $\beta$ & Worst $\beta$ & $\operatorname{Avg} \beta$ & $\operatorname{Std} \beta$ & Iteration & Avg Iteration & Std Iteration \\
\hline \multirow{5}{*}{$g_{1}$} & $\mathrm{ICDE}$ & 2.9057 & 3.0942 & 2.9151 & 0.0421 & 34 & 38.35 & 4.88 \\
\hline & IRO & 2.9058 & 3.1025 & 2.9946 & 0.0957 & 100 & 105.80 & 27.29 \\
\hline & DPSO & 2.9056 & 3.1430 & 2.9881 & 0.0990 & 52 & 121.25 & 36.51 \\
\hline & $\mathrm{CBO}$ & 2.9057 & 3.5352 & 3.0307 & 0.1768 & 38 & 39.75 & 14.02 \\
\hline & ECBO & 2.9056 & 3.0951 & 2.9274 & 0.0573 & 190 & 118.85 & 50.78 \\
\hline \multirow{5}{*}{$g_{2}$} & ICDE & 2.9999 & 2.9999 & 2.9999 & 0.0000 & 31 & 32.00 & 1.00 \\
\hline & IRO & 2.9999 & 3.0005 & 2.9999 & 0.0001 & 66 & 71.30 & 13.43 \\
\hline & DPSO & 2.9999 & 2.9999 & 2.9999 & $5.17 \mathrm{E}-07$ & 36 & 40.60 & 7.80 \\
\hline & CBO & 2.9999 & 3.0053 & 3.0001 & 0.0012 & 20 & 27.15 & 7.67 \\
\hline & ECBO & 2.9999 & 3.0237 & 3.0020 & 0.0057 & 149 & 127.15 & 47.85 \\
\hline \multirow{5}{*}{$g_{3}$} & ICDE & 1.9999 & 1.9999 & 1.9999 & 0.0000 & 32 & 38.00 & 5.00 \\
\hline & IRO & 1.9999 & 2.0059 & 2.0006 & 0.0016 & 47 & 93.20 & 34.20 \\
\hline & DPSO & 1.9999 & 2.0000 & 1.9999 & $3.12 \mathrm{E}-05$ & 63 & 60.80 & 22.42 \\
\hline & $\mathrm{CBO}$ & 1.9999 & 2.0004 & 1.9999 & 0.000126 & 26 & 27.70 & 8.49 \\
\hline & ECBO & 1.9999 & 2.113 & 2.0056 & 0.0252 & 43 & 68.65 & 37.48 \\
\hline \multirow{5}{*}{$g_{4}$} & ICDE & 2.4999 & 2.4999 & 2.4999 & 0.0000 & 32 & 41.00 & 17.00 \\
\hline & IRO & 2.4999 & 2.5013 & 2.5000 & 0.0003 & 67 & 78.05 & 28.99 \\
\hline & DPSO & 2.4999 & 2.5012 & 2.5001 & 0.0002 & 32 & 64.25 & 31.65 \\
\hline & CBO & 2.4999 & 2.5256 & 2.5031 & 0.0057 & 39 & 27.05 & 7.50 \\
\hline & ECBO & 2.4999 & 2.5149 & 2.5009 & 0.0033 & 19 & 55.10 & 61.32 \\
\hline \multirow{5}{*}{$g_{5}$} & ICDE & 1.6583 & 1.6583 & 1.6583 & 0.0000 & 36 & 41.00 & 4.00 \\
\hline & IRO & 1.6582 & 1.6679 & 1.6595 & 0.0022 & 98 & 96.25 & 16.91 \\
\hline & DPSO & 1.6582 & 1.6617 & 1.6589 & 0.001 & 42 & 84.80 & 41.60 \\
\hline & $\mathrm{CBO}$ & 1.6582 & 1.7081 & 1.6663 & 0.0122 & 20 & 36.05 & 9.58 \\
\hline & ECBO & 1.6583 & 1.6609 & 1.6588 & 0.0005 & 73 & 78.35 & 47.29 \\
\hline \multirow{5}{*}{$g_{6}$} & ICDE & 2.7099 & 2.7099 & 2.7099 & 0.0000 & 35 & 32.00 & 2.00 \\
\hline & IRO & 2.7129 & 6.3233 & 3.5772 & 1.1430 & 162 & 132.35 & 24.20 \\
\hline & DPSO & 2.7103 & 5.4257 & 3.7728 & 0.8326 & 88 & 127.70 & 40.97 \\
\hline & CBO & 2.7109 & 3.9674 & 3.0350 & 0.3724 & 47 & 51.55 & 12.22 \\
\hline & ECBO & 2.7149 & 5.6122 & 3.2572 & 0.6505 & 118 & 118.3 & 37.95 \\
\hline \multirow{5}{*}{$g_{7}$} & ICDE & 3.3496 & 3.3496 & 3.3496 & 0.0000 & 32 & 35.00 & 3.00 \\
\hline & IRO & 3.3496 & 3.3519 & 3.3500 & 0.0005 & 90 & 82.50 & 19.18 \\
\hline & DPSO & 3.3496 & 3.3653 & 3.3522 & 0.0041 & 109 & 108.75 & 53.06 \\
\hline & $\mathrm{CBO}$ & 3.3496 & 3.556 & 3.367 & 0.0479 & 30 & 27.40 & 10.049 \\
\hline & ECBO & 3.3496 & 3.362 & 3.3513 & 0.0029 & 54 & 99.25 & 52.35 \\
\hline \multirow{5}{*}{$g_{8}$} & ICDE & 1.9990 & 6.980 & 3.1302 & 1.5801 & 191 & 181.20 & 31.23 \\
\hline & IRO & 2.0053 & 2.1244 & 2.0293 & 0.0271 & 185 & 174.15 & 20.38 \\
\hline & DPSO & 2.0000 & 2.0045 & 2.0009 & 0.0010 & 111 & 137.55 & 16.86 \\
\hline & CBO & 1.9999 & 6.9571 & 3.0399 & 1.5502 & 128 & 157.95 & 30.80 \\
\hline & ECBO & 2.0000 & 6.2084 & 2.2107 & 0.9409 & 121 & 132.00 & 25.06 \\
\hline \multirow{5}{*}{$g_{9}$} & ICDE & 2.2696 & 7.9703 & 3.4893 & 1.555 & 108 & 160.55 & 31.08 \\
\hline & IRO & 2.2769 & 5.1388 & 3.2534 & 0.9307 & 170 & 163.05 & 18.77 \\
\hline & DPSO & 2.3413 & 5.9199 & 3.2534 & 1.0863 & 31 & 125.75 & 46.21 \\
\hline & $\mathrm{CBO}$ & 2.2701 & 4.6905 & 2.5852 & 0.5750 & 43 & 60.60 & 11.45 \\
\hline & ECBO & 2.3814 & 5.0723 & 3.2628 & 0.8835 & 88 & 121.80 & 40.29 \\
\hline \multirow{5}{*}{$g_{10}$} & ICDE & 5.3333 & 5.3336 & 5.3334 & 0.0001 & 39 & 46.00 & 5.00 \\
\hline & IRO & 5.3370 & 5.4181 & 5.3568 & 0.0261 & 57 & 90.80 & 42.37 \\
\hline & DPSO & 5.3516 & 5.4278 & 5.3946 & 0.0256 & 117 & 110.00 & 46.67 \\
\hline & CBO & 5.3442 & 5.4277 & 5.4008 & 0.0247 & 46 & 55.60 & 14.46 \\
\hline & ECBO & 5.3387 & 5.4280 & 5.3878 & 0.0317 & 53 & 104.90 & 42.49 \\
\hline \multirow{5}{*}{$g_{11}$} & $\mathrm{ICDE}$ & 2.4999 & 2.4999 & 2.4999 & 0.0000 & 30 & 32.00 & 2.00 \\
\hline & IRO & 2.4999 & 2.5005 & 2.5000 & 0.0001 & 60 & 67.65 & 16.66 \\
\hline & DPSO & 2.4999 & 2.5009 & 2.5001 & 0.0003 & 38 & 54.95 & 24.20 \\
\hline & $\mathrm{CBO}$ & 2.4999 & 2.5149 & 2.5015 & 0.0035 & 23 & 25.30 & 6.58 \\
\hline & ECBO & 2.4999 & 2.5038 & 2.5009 & 0.0011 & 30 & 63.70 & 55.28 \\
\hline
\end{tabular}


For the limit state function $\boldsymbol{g}_{1}$, the convergence history of the mean values of the reliability index over 20 runs of the ICDE and those are available in the literature are presented in Fig. 3. The population size and maximum number of iteration are set 20 and 200 respectively. As can be observed, the ICDE converges to the optimum value faster than the others.

\subsection{Example 2: limit state function with non-normal basic variables}

In order to test the efficiency of ICDE in the problems with non-normal basic variables, the non-linear noisy limit state function is considered. The function is [30]:

$$
g=x_{1}+2 x_{2}+2 x_{3}+x_{4}-5 x_{5}-5 x_{6}+100 \sum_{i=1}^{6} \sin \left(100 x_{i}\right)
$$

As shown in in Table 5, all six random variables are log-normally distributed. The computational results obtained with different methods are presented in Table 6 , where $\mathrm{x}^{*}$ is the MPP in the original space. It is seen that ICDE converges to a better reliability index than the other methods.

Table 5 Random variables for the noisy limit state function in example 2

\begin{tabular}{lccc}
\hline Variables & Distribution & $\mu$ & $\sigma$ \\
\hline $\boldsymbol{x}_{1}, \boldsymbol{x}_{2}, \boldsymbol{x}_{3}, \boldsymbol{x}_{4}$ & Log-normal & 120 & 12 \\
$\boldsymbol{x}_{5}$ & Log-normal & 50 & 15 \\
$\boldsymbol{x}_{6}$ & Log-normal & 40 & 12 \\
\hline
\end{tabular}

Table 6 Results for the noisy limit state function in example 2

\begin{tabular}{ccccc}
\hline & ICDE & Hybrid GA[15] & MCS [15] & ARBIS [29] \\
\hline$\beta$ & 2.348139 & 2.615 & 2.614 & 2.361 \\
$P_{f}$ & 0.00943 & - & - & - \\
& $(117.29,115.22$, & & & - \\
$\mathrm{X}^{*}$ & $\begin{array}{c}115.21,117.22, \\
\end{array}$ & - & - & \\
\hline
\end{tabular}

The ICDE method, for all the previous problems with different basic variables distributions, is proved to be very reliable and accurate. In the next section, the Application of the method in structural reliability problems will be considered.

\subsection{Example 3: structural applications}

In this section, the application of the ICDE is proposed in six structural reliability problems and the results are compared with those are available in the previous studies.

\subsubsection{Cantilever beam with uniformly distributed load problem}

Consider a simple cantilever beam of length $L$ with rectangular cross section subject to a uniformly distributed loading as shown in Fig. 4. The limit state function according to the maximum deflection at the free end is defined as follows [31]:

$$
(w, b, E, I, L)=-\frac{(w \times b) L^{4}}{8 E I}+\frac{L}{325}
$$

Where $w, b, E$ and $I$ are the load per unit area, width, Young's modulus and area moment of the cross section respectively. $E$ and $L$ are assumed to be the deterministic variables and equal to $2.6 \times 10^{4} \mathrm{Mpa}$ and $6 \mathrm{~m}$ respectively. So, the limit state function becomes:

$$
g(\boldsymbol{x})=18.46154-7.476923 \times 10^{10} \frac{x_{1}}{x_{2}^{3}}
$$

Where $x_{1}$ and $x_{2}$ are respectively the load and depth. These two variables are the independent normal random variables with $\mu_{x_{1}}=0.001 \mathrm{MPa}, \mu_{x_{2}}=250 \mathrm{~mm}$ and $\sigma_{x_{1}}=0.0002, \sigma_{x_{2}}=37.5$;

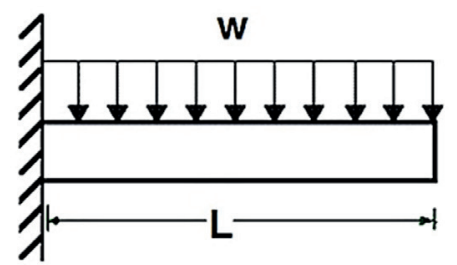

Fig. 4 Cantilever beam with uniformly distributed load

The results are proposed in Table 7. It can be seen that all metaheuristic algorithms converge to almost the same reliability index value. The statistical results in Table 8 show that the ICDE has better results than those are available in the literature in terms of the worst $\beta, \operatorname{avg} \beta, \operatorname{std} \beta$, iteration, avg iteration and std iteration.

The convergence curves over 20 runs of the ICDE, IRO, DPSO, ECBO and a single run for the CPSO are proposed in Fig. 5. Whilst the population size and maximum number of iteration for these algorithms are considered 20 and 200 respectively, those of CPSO are 1000 and 100 respectively. It can be observed that the convergence rate of the ICDE is superior compared to the other algorithms. It is noted that using the CPSO the reliability index is converged after 50 iterations [17].

\subsubsection{Conical structure problem}

Consider a conical structure under a compressive axial load $P$, and a bending moment $M$. The geometrical configuration and random variables are shown in Fig. 6 and Table 9 respectively.

The loss of strength and buckling of the structure are the two main failure modes that can cause the instability of the structure. The first one is not considered due to the large margin obtained in the analysis [4]. So only the buckling mode will be analyzed under the combined solicitations. The buckling criterion is defined as follows:

$$
\frac{P}{P_{\text {crit }}}+\frac{M}{M_{\text {crit }}} \geq 1
$$

Where $P_{c r i t}$ and $M_{c r i t}$ are respectively the critical axial load and bending moment, which are defined by NASA [32]:

$$
P_{c r i t}=\gamma \frac{2 \pi E t^{2} \cos ^{2} \alpha}{\sqrt{3\left(1-\mu^{2}\right)}}
$$


Table 7 Results for the cantilever beam with distributed load problem in example 3

\begin{tabular}{|c|c|c|c|c|c|c|c|c|c|c|c|}
\hline & ICDE & IRO[18] & DPSO[18] & $\mathrm{CBO}[18]$ & $\mathrm{ECBO}[18]$ & CSS [5] & PSO [4] & ICA [16] & iBA[6] & CPSO[17] & $\operatorname{MCS}[17]$ \\
\hline$\beta$ & 2.3309 & 2.3309 & 2.3309 & 2.3309 & 2.3310 & 2.3309 & 2.3309 & 2.3309 & 2.3326 & 2.3312 & - \\
\hline$P_{f}$ & 0.00988 & 0.00988 & 0.00988 & 0.00988 & 0.00988 & 0.00988 & 0.00988 & - & 0.00980 & 0.00987 & 0.96070 \\
\hline$x_{1}$ & 0.00112 & 0.00111 & 0.00111 & 0.00111 & 20.00111 & 0.00100 & 0.00119 & 0.00112 & 0.00110 & 0.00113 & - \\
\hline$x_{2}$ & 165.449 & 165.480 & 165.480 & 165.530 & 165.240 & 159.399 & 165.437 & 165.451 & 166.360 & 165.810 & - \\
\hline
\end{tabular}

Table 8 Statistical results obtained by different methods for the cantilever beam problem in example 3

\begin{tabular}{|c|c|c|c|c|c|c|c|}
\hline & Best $\beta$ & Worst $\beta$ & Avg $\beta$ & $\operatorname{Std} \beta$ & Iteration & Avg Iteration & Std Iteration \\
\hline ICDE & 2.3309 & 2.3309 & 2.3309 & 0.0000 & 32 & 36.00 & 3.000 \\
\hline IRO [18] & 2.3309 & 2.4651 & 2.3546 & 0.0320 & 85 & 126.15 & 30.906 \\
\hline DPSO [18] & 2.3309 & 2.4613 & 2.3497 & 0.0346 & 67 & 139.35 & 49.9634 \\
\hline CBO [18] & 2.3309 & 2.6532 & 2.3614 & 0.0813 & 33 & 38.30 & 13.5805 \\
\hline ECBO [18] & 2.3310 & 2.4026 & 2.3447 & 0.0193 & 76 & 117.20 & 38.7292 \\
\hline
\end{tabular}

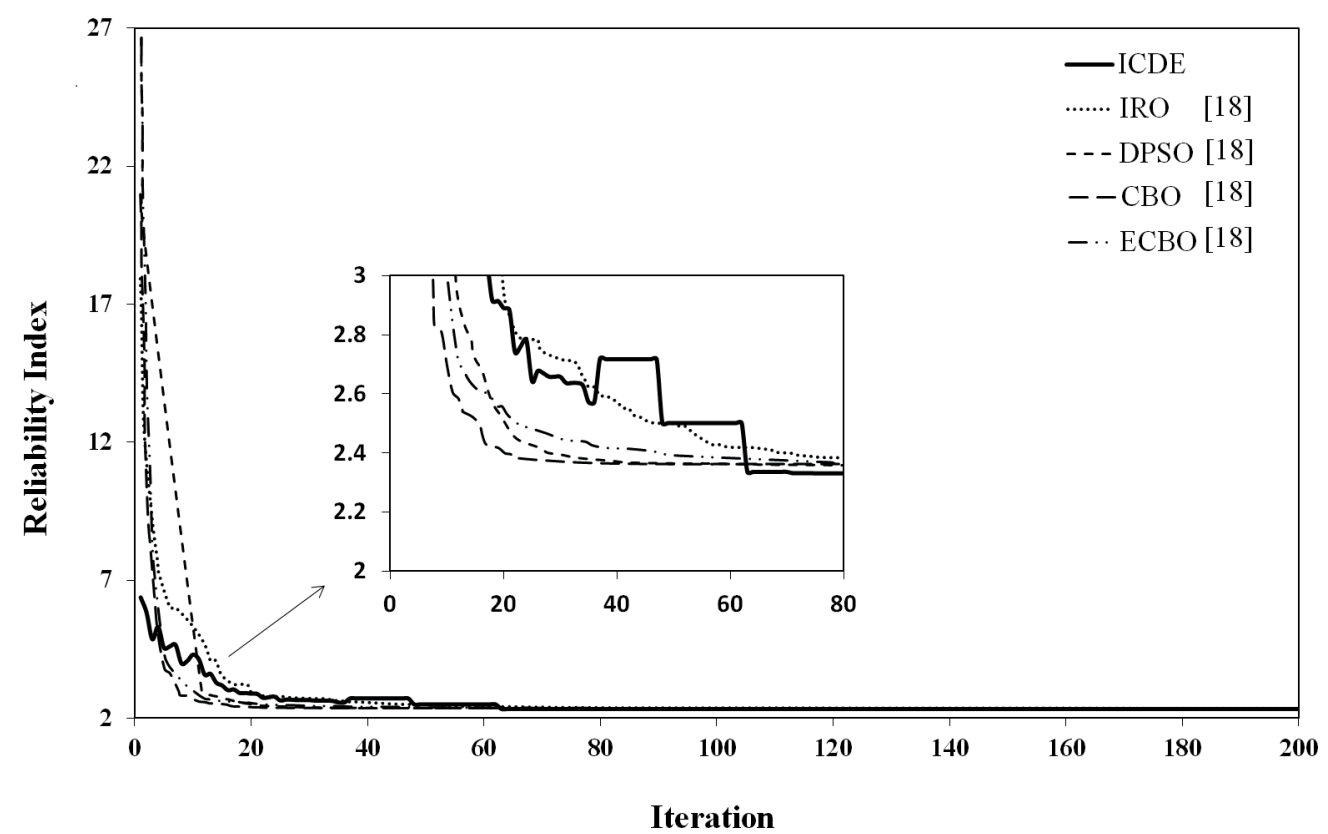

Fig. 5 Comparison of the average values of the reliability index obtained from the different algorithms for cantilever beam problem

$$
M_{c r i t}=\eta \frac{2 \pi E t^{2} r_{1} \cos ^{2} \alpha}{\sqrt{3\left(1-\mu^{2}\right)}}
$$

Where $\gamma=0.33, \eta=0.41$ and $\mu=0.334$. From equations (17), (18) and (19), the limit state function is:

$$
g(\boldsymbol{x})=1-\frac{\sqrt{3\left(1-\mu^{2}\right)}}{\pi x_{1} x_{2}^{2} \cos ^{2} x_{3}}\left(\frac{x_{6}}{2 \gamma}+\frac{x_{5}}{\eta x_{4}}\right)
$$

Table 9 Random variables for the conical structure problem in example 3

\begin{tabular}{lcccc}
\hline & Variables & Distribution & $\mu$ & $\sigma$ \\
\hline $\boldsymbol{x}_{1}$ & $E(\mathrm{MPa})$ & Normal & 70000 & 3500 \\
$\boldsymbol{x}_{2}$ & $t(\mathrm{~m})$ & Normal & 0.0025 & 0.000125 \\
$\boldsymbol{x}_{3}$ & $\alpha(\mathrm{rad})$ & Normal & 0.5240 & 0.010480 \\
$\boldsymbol{x}_{4}$ & $r_{1}(\mathrm{~m})$ & Normal & 0.9000 & 0.022500 \\
$\boldsymbol{x}_{5}$ & $M(\mathrm{~N} . \mathrm{m})$ & Normal & 80000 & 6400 \\
$\boldsymbol{x}_{6}$ & $P(\mathrm{~N})$ & Normal & 70000 & 5600 \\
\hline
\end{tabular}

The optimum design variables, reliability index and the probability of failure are presented in Table 10. It can be observed that the ICDE converges to a better Reliability index than the other metaheuristic methods. From the statistical results in Table 11, the ICDE has better performance in terms of the best $\beta, \operatorname{avg} \beta$ and iteration.

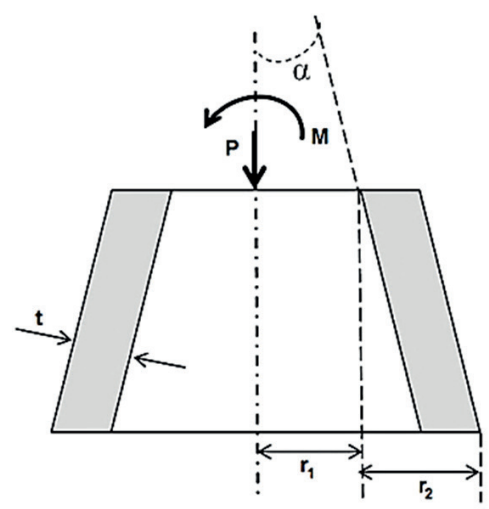

Fig. 6 Geometrical configuration of the conical structure 
Table 10 Results for conical structure problem in example 3

\begin{tabular}{lccccccccc}
\hline & ICDE & IRO [18] & DPSO [18] & CBO [18] & ECBO [18] & CSS [5] & PSO [4] & iBA [6] & HL-RF [6] \\
\hline$\beta$ & 4.7959 & 4.7964 & 4.7970 & 4.7995 & 4.7989 & 4.7965 & 4.8830 & 4.8770 & 4.8770 \\
$P_{f}$ & $8.10 \mathrm{E}-07$ & $8.07 \mathrm{E}-07$ & $0.8 .05 \mathrm{e}-7$ & $7.95 \mathrm{E}-07$ & $7.97 \mathrm{E}-07$ & $8.07 \mathrm{E}-07$ & $5.23 \mathrm{E}-07$ & $5.12 \mathrm{E}-07$ & $5.12 \mathrm{E}-07$ \\
$\boldsymbol{x}_{1}$ & $6.38 \mathrm{E}+10$ & $6.38 \mathrm{E}+10$ & $6.39 \mathrm{E}+10$ & $6.42 \mathrm{E}+10$ & $6.39 \mathrm{E}+10$ & $6.38 \mathrm{E}+10$ & $6.37 \mathrm{E}+10$ & $6.37 \mathrm{E}+10$ & $6.37 \mathrm{E}+10$ \\
$\boldsymbol{x}_{2}$ & 0.00199 & 0.00199 & 0.00199 & 0.00199 & 0.00199 & 0.00199 & 0.00198 & 0.00199 & 0.00199 \\
$\boldsymbol{x}_{3}$ & 0.52812 & 0.52789 & 0.52788 & 0.52867 & 0.52888 & 0.52813 & 0.52446 & 0.52810 & 0.52820 \\
$\boldsymbol{x}_{4}$ & 0.88730 & 0.88833 & 0.88580 & 0.88620 & 0.88624 & 0.88734 & 0.88743 & 0.88730 & 0.88720 \\
$\boldsymbol{x}_{5}$ & 90103.55 & 90364.00 & 90136.00 & 90752.00 & 90883.00 & 90081.00 & 90295.20 & 90258.80 & 90208.20 \\
$\boldsymbol{x}_{6}$ & 74243.28 & 74181.00 & 74424.00 & 74333.00 & 73990.00 & 74255.50 & 131496.39 & 74308.70 & 74307.40 \\
\hline
\end{tabular}

Table 11 Statistical results obtained by different methods for the conical structure problem in example 3

\begin{tabular}{|c|c|c|c|c|c|c|c|}
\hline & Best $\beta$ & Worst $\beta$ & $\operatorname{Avg} \beta$ & $\operatorname{Std} \beta$ & Iteration & Avg Iteration & Std Iteration \\
\hline ICDE & 4.7959 & 4.8612 & 4.7924 & 0.0147 & 47 & 168.0 & 57.0 \\
\hline IRO [18] & 4.7963 & 4.8038 & 4.7986 & 0.0021 & 85 & 133.1 & 38.7 \\
\hline DPSO [18] & 4.7970 & 4.8100 & 4.8001 & 0.0033 & 152 & 123.4 & 48.3 \\
\hline CBO [18] & 4.7995 & 5.1449 & 4.8548 & 0.0805 & 77 & 91.1 & 23.5 \\
\hline ECBO [18] & 4.7989 & 4.8434 & 4.8139 & 0.0119 & 141 & 161.5 & 23.8 \\
\hline
\end{tabular}

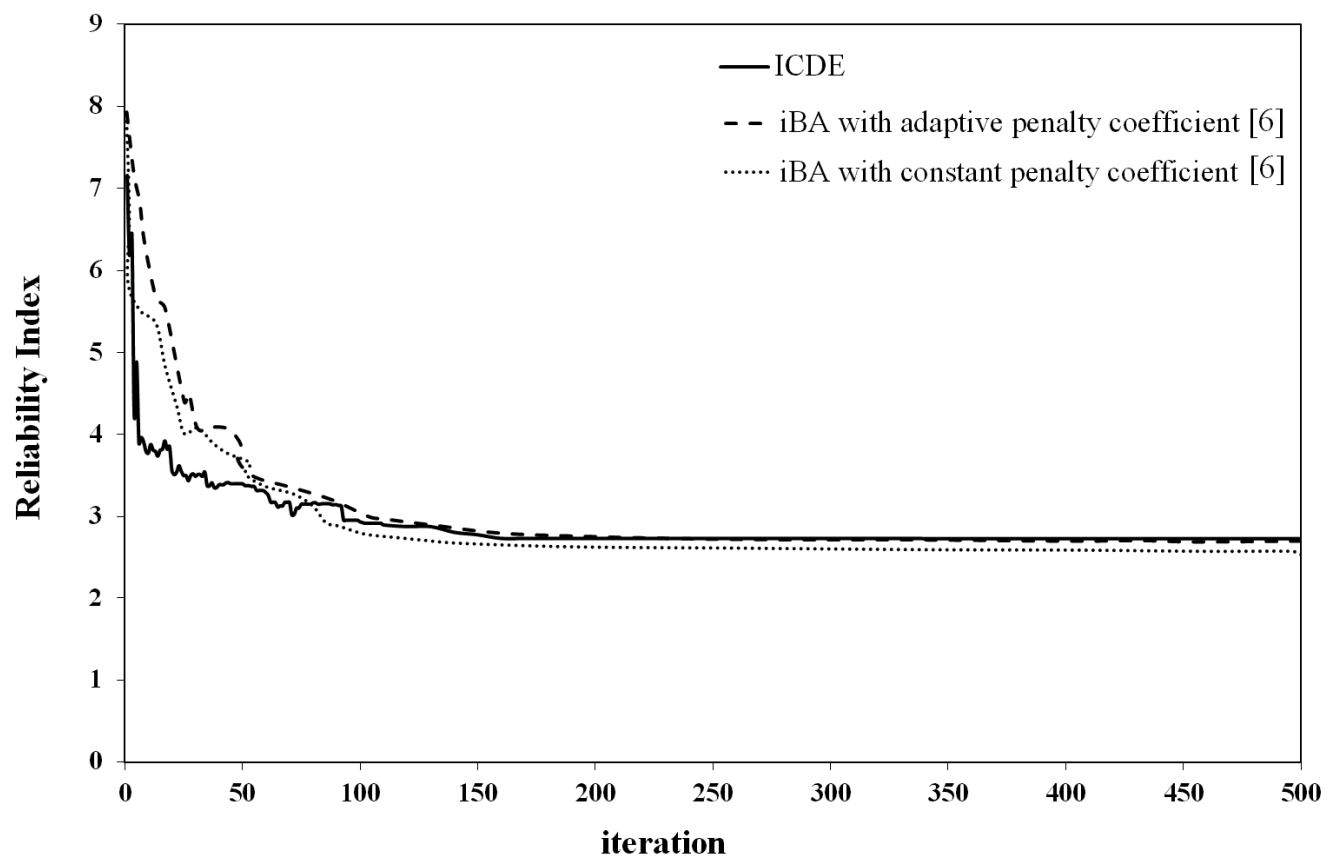

Fig. 7 Comparison of the average values of the reliability index obtained from the ICDE and iBA algorithms for the reinforced concrete beam problem

\subsubsection{Reinforced concrete beam problem}

In this example, the reliability analysis of a reinforced concrete beam is performed. The limit state function is defined as follows $[6,33]$ :

$$
g\left(A_{s}, F_{y}, F_{c}, Q\right)=A_{s} F_{y} d-\frac{0.59\left(A_{s} F_{y}\right)^{2}}{F_{c} b}
$$

Where $A_{s}, F_{v}$, and $Q$ are respectively the reinforcing cross-section area, the yield strength of the reinforced steel rods, the compressive strength of concrete and the total moment produced by the load, Theses random variables are described in Table 12. The parameters $\mathrm{b}$ and $\mathrm{d}$ are width and height respectively ( $b=12$ in, $d=19$ in).
Table 12 Random variables for the reinforced concrete beam problem in example 3

\begin{tabular}{lcccc}
\hline & Variables & Distribution & $\mu$ & $\sigma$ \\
\hline $\boldsymbol{x}_{1}$ & $A_{s}(\mathrm{in} 2)$ & Normal & 4.08 & 0.0816 \\
$\boldsymbol{x}_{2}$ & $F_{y}(\mathrm{ksi})$ & Normal & 44.00 & 4.6200 \\
$\boldsymbol{x}_{3}$ & $F_{c}(\mathrm{ksi})$ & Normal & 3.12 & 0.4368 \\
$\boldsymbol{x}_{4}$ & $Q$ (kip-in) & Normal & 2052.00 & 246.24 \\
\hline
\end{tabular}

According to the results which are shown in Table 13, the performance of the ICDE is superior compared to the other metaheuristic algorithms in the sense of the reliability index. However, the results of the ICDE and HL-RF are almost the same. Compared with the Monte Carlo Simulation (MSC) approach, the ICDE is less accurate but it requires the less computational cost to find an appropriate design point. 
Table 13 Results for the reinforced concrete beam problem in example 3

\begin{tabular}{|c|c|c|c|c|c|c|c|c|}
\hline & ICDE & iBA[6] & $\mathrm{BA}[6]$ & PSO [6] & HS [6] & GA[6] & HL-RF [6] & $\operatorname{MCS}$ [33] \\
\hline$\beta$ & 2.3363 & 2.3493 & 2.7493 & 2.3668 & 8.1267 & 2.9222 & 2.3350 & 2.2900 \\
\hline$P_{f}$ & 0.0097 & 0.0094 & 0.0030 & 0.0090 & $2.206 \mathrm{e}-16$ & 0.0017 & 0.0098 & 0.0110 \\
\hline$x_{1}$ & 36.022 & 35.610 & - & - & - & - & 36.230 & 35.500 \\
\hline$x_{2}$ & 4.059 & 4.047 & - & - & - & - & 4.056 & 4.000 \\
\hline$x_{3}$ & 3.022 & 2.976 & - & - & - & - & 2.970 & 3.110 \\
\hline$x_{4}$ & 2430.02 & 2395.12 & - & - & - & - & 2436.00 & 2408.50 \\
\hline
\end{tabular}

Since the efficiency of iBA is superior compared to the standard algorithms such as GA, PSO, HS and BA [6], only the convergence histories of the ICDE and iBA are compared in Fig. 7. Each algorithm was run 100 times with a population size of 100 . It can be seen that the convergence of iBA can be significantly dependent on the choice of the penalty coefficient. But the ICDE converges to an appropriate value of reliability index without the need to perform fine-tuning of the parameters related to the handling of the constraint. In the next three sections, the structural problems containing non-normal distributed variables are considered.

\subsubsection{Cantilever beam with concentrated load problem}

A cantilever steel beam with span $L$ and rectangular crosssection subjected to a concentrated load $P$ at the end is studied in this example. The limit state function is [5]:

$$
g\left(Z, F_{y}, P\right)=Z F_{y}-P L
$$

Where $Z$ is section modulus and $F_{y}$ is the yield stress. The random variables are defined in Table 14 , and $L$ is equal to $2(\mathrm{~m})$.

Table 14 Random variables for the cantilever beam with concentrated load in example 3

\begin{tabular}{lcccc}
\hline & Variables & Distribution & $\mu$ & $\sigma$ \\
\hline $\boldsymbol{x}_{1}$ & $F_{y}\left(\mathrm{KN} / \mathrm{mm}^{2}\right)$ & Normal & 0.32 & 0.032 \\
$\boldsymbol{x}_{2}$ & $Z\left(\mathrm{~mm}^{3}\right)$ & Normal & $1.4 \times 10^{6}$ & $70 \times 10^{3}$ \\
$\boldsymbol{x}_{3}$ & $P(\mathrm{KN})$ & Log-normal & 100 & 100 \\
\hline
\end{tabular}

The final results are presented in Table 15. It is observed that the optimal points obtained by ICDE, CSS and HL-RF are very similar.

Table 15 Results for the cantilever beam with concentrated load problem in

\begin{tabular}{lccc}
\hline & \multicolumn{3}{c}{ example 3 } \\
\hline$\beta$ & ICDE & CSS [5] & HL-RF [34] \\
$P_{f}$ & 0.01422 & 2.191 & 2.192 \\
$\boldsymbol{x}_{1}$ & 0.30153 & 0.0142 & 0.0142 \\
$\boldsymbol{x}_{2}$ & $1.38 \mathrm{e}+06$ & 0.30152 & 0.3149 \\
$\boldsymbol{x}_{3}$ & 208.1585 & $1.38 \mathrm{e}+06$ & $1.38 \mathrm{e}+06$ \\
\hline
\end{tabular}

\subsubsection{Tower of a cable-stayed bridge problem}

In this example, the behavior of a tower of a cable-stayed bridge constructed in France in 1977 along the longitudinal direction is studied [35]. As shown in Fig. 8, the tower is subjected to a coupled axial force and moment.

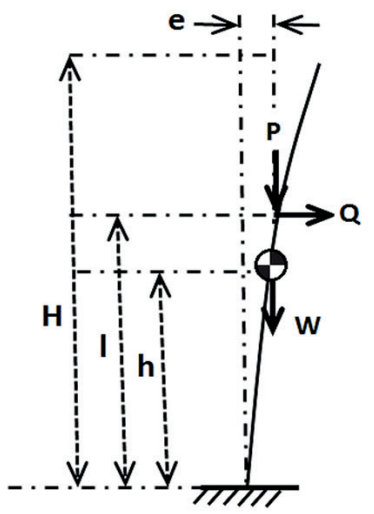

Fig. 8 Model of the tower of a cable-stayed bridge

The limit state function of the bridge tower's bending resistance is defined as:

$$
g=M-P e-W\left(\frac{h}{l}\right)^{2} e-Q l
$$

$M, W, P$ and $Q$ are the moment resistance of a section at the bridge tower foot, deadweight of the tower, vertical and horizontal components of the forces of all stay cables, respectively. $L$ and $H$ are the height of the resultant force of the stay cables and the height of the bridge tower, respectively, and $h$ is the height of the center of gravity of the tower which is equal to $0.4 \mathrm{H}$. The eccentricity, e, is expressed as:

$$
g=\frac{Q l^{3}}{3 E I\left(1-\frac{2}{5} \frac{P l^{2}}{E I}\right)}
$$

Where $E$ is the elastic modulus of the bridge tower and $I$ is equivalent inertia moment.

Furthermore, an additional moment may be occurred by vehicle loads and temperature variation. Here, this additional moment is equivalent to an additive horizontal force $Q^{\prime}$. By replacing $Q$ with $Q^{\prime}$ in equation (23)

$$
g=M-\frac{P Q^{\prime} l^{3}}{3 E I\left(1-\frac{2}{5} \frac{P l^{2}}{E I}\right)}-\frac{W Q^{\prime} l h^{2}}{3 E I\left(1-\frac{2}{5} \frac{P l^{2}}{E I}\right)}-Q^{\prime} l
$$


The parameters $H, l, W$ and $h$ are equal to $70.5 \mathrm{~m}, 47.4 \mathrm{~m}$, $1170 \mathrm{kN}$ and $28.2 \mathrm{~m}$, respectively. $I, E, P, Q^{\prime}$, and $M$ are random variables which are listed in Table 16.

Table 16 Random variables for the tower of a cable-stayed bridge problem in example 3

\begin{tabular}{lcccc}
\hline & Variables & Distribution & $\mu$ & $\sigma$ \\
\hline $\boldsymbol{x}_{1}$ & $I\left(\mathrm{~m}^{4}\right)$ & Normal & 17.6 & 1.76 \\
$\boldsymbol{x}_{2}$ & $E\left(\mathrm{kN} / \mathrm{m}^{2}\right)$ & Log-normal & $4 \times 10^{7}$ & $3.210^{6}$ \\
$\boldsymbol{x}_{3}$ & $P(\mathrm{kN})$ & Normal & $9.01 \times 10^{4}$ & $9.01 \times 10^{3}$ \\
$\boldsymbol{x}_{4}$ & $Q^{\prime}(\mathrm{kN})$ & Normal & $2.12 \times 10^{3}$ & $3.18 \times 10^{2}$ \\
$\boldsymbol{x}_{5}$ & $M(\mathrm{kN} . \mathrm{m})$ & Normal & $2.6 \times 10^{5}$ & $3.09 \times 10^{4}$ \\
\hline
\end{tabular}

For comparison, the results by ICDE and PSO based on the preference of the feasible solutions technique are provided in Table 17. It is seen that The ICDE results have a good agreement with the literature.

Table 17 Results for the tower of a cable-stayed bridge problem in example 3

\begin{tabular}{lcc}
\hline & ICDE & PSO [35] \\
\hline$\beta$ & 3.4946 & 3.4950 \\
$P_{f}$ & 0.000237 & 0.0002 \\
$\boldsymbol{x}_{1}$ & 17.3713245 & 17.3711 \\
$\boldsymbol{x}_{2}$ & $3.9548 \mathrm{E}+07$ & $3.9547 \mathrm{E}+07$ \\
$\boldsymbol{x}_{3}$ & $9.1237 \mathrm{E}+04$ & $9.1238 \mathrm{E}+04$ \\
$\boldsymbol{x}_{4}$ & $2.5585 \mathrm{E}+03$ & $2.5585 \mathrm{E}+03$ \\
$\boldsymbol{x}_{5}$ & $1.3503 \mathrm{E}+05$ & $1.3503 \mathrm{E}+05$ \\
\hline
\end{tabular}

\subsubsection{Steel joint problem}

The last example considers a highly nonlinear limit state function which is related to the stress distribution in a steel joint addressing elevated temperature and phenomena of fatigue. The limit state function is defined as [36]:

$$
g(\boldsymbol{x})=x_{1}-10^{4}\left(\frac{x_{2}\left(x_{4} x_{5}\right)^{1.71}}{x_{3}}-\frac{\left(1-x_{2}\right)\left(x_{4} x_{5}\right)^{1.88}}{x_{6}}\right)
$$

The random variables are listed in Table 18. For comparison purposes, the results by ICDE, iBA, Adaptive RSM (Response Surface Method) and RSM are provided in Table 19. It is observed that ICDE converges to those based on the sampling methods.

Table 18 Random variables for the steel joint problem in example 3

\begin{tabular}{lccc}
\hline & Distribution & $\mu$ & $\sigma$ \\
\hline $\boldsymbol{x}_{1}$ & Log-normal & 1.0440 & 0.31320 \\
$\boldsymbol{x}_{2}$ & Normal & 0.7000 & 0.07000 \\
$\boldsymbol{x}_{3}$ & Log-normal & 0.2391 & 0.09564 \\
$\boldsymbol{x}_{4}$ & Log-normal & 1.011 & 0.15165 \\
$\boldsymbol{x}_{5}$ & Gumbel max & 0.0005 & 0.0008 \\
$\boldsymbol{x}_{6}$ & Log-normal & 1.802 & 0.7208 \\
\hline
\end{tabular}

\section{Conclusions}

In this paper, the first order reliability method is used to calculate the probability of failure. Since finding the MPP is a constrained optimization problem, in contrast to all the previous studies based on the penalty function method or the preference of the feasible solutions technique, in this study, the ICDE metaheuristic algorithm based on the multi-objective constraint-handling technique is utilized. Several examples are used to investigate the accuracy and efficiency of the ICDE. The optimum reliability indexes obtained by the ICDE are approximately identical to those yielded by the MCS in the literature, so the accuracy of the algorithm is verified. The comparison between the ICDE and the other metaheuristic algorithms based on the penalty function method shows that it has a reliable and acceptable performance. Based on the results, ICDE shows faster convergence rate than IRO, DPSO, $\mathrm{CBO}$ and $\mathrm{ECBO}$ in the process of finding the design point. Also, it can be seen from the statistical results that the performance of the ICDE is superior to or at least quite competitive with the all other methods for all considered problems. In addition, the ICDE is very easy to implement because there is no need to the time-consuming task of fine tuning of the penalty parameters. According to the relatively fast convergence rate, low standard deviation from the mean value of the optimum reliability index and ease of implementation, it can be expected that the proposed method would be a robust alternative to the penalty function based approaches for the reliability assessment problems in the future works.

\section{References}

[1] Ang, A. H., Tang, W. H. "Probability Concepts in Engineering: Emphasis on Applications to Civil and Environmental Engineering". Structure and Infrastructure Engineering, 4(5), pp. 413-414. 2008. https://doi. org/10.1080/15732470802027894

[2] Haldar, A., Mahadevan, S. "Probability, reliability, and statistical methods in engineering design". John Wiley \& Sons, New York, 2000. https://doi.org/10.1002/bate.200002930

[3] Madsen, H. O., Krenk, S., Lind, N. C. "Methods of structural safety". Prentice-Hall, Englewood Cliffs, New Jersey, 1986.

[4] Elegbede, C. "Structural reliability assessment based on particles swarm optimization". Structural Safety, 27(2), pp. 171-186, 2005. https://doi. org/10.1016/j.strusafe.2004.10.003 
[5] Kaveh, A., Massoudi, M., Ghanooni Bagha, M. "Structural reliability analysis using charged system search algorithm". Iranian Journal of Science and Technology, 38(C2), pp. 439-448. 2014. http://ijstc.shirazu. ac.ir/article_2420_4bebe981b7a6d56f6b5d81b9621ffe19.pdf

[6] Chakri, A., Khelif, R., Benouaret, M. "Improved bat algorithm for structural reliability assessment: application and challenges". Multidiscipline Modeling in Materials and Structures, 12(2), pp. 218-253, 2016. https://doi.org/10.1108/mmms-07-2015-0035

[7] Choi, S.-K., Canfield, R.A. and Grandhi, R.V. "Reliability-based Structural Design". Springer-Verlag, 2007. https://doi.org/10.1007/978-184628-445-8

[8] Chiralaksanakul, A., Mahadevan, S. "First-Order Approximation Methods in Reliability-Based Design Optimization". Journal of Mechanical Design, 127(5), pp. 851-857, 2004. https://doi.org/10.1115/1.1899691

[9] Hasofer, A. M., Lind, M. C. "An exact and invariant first order reliability format". Journal of Engineering Mechanics, 100, pp. 111-12. 1974.

[10] Hasofer, A. M., Lind, N.C. "Exact and invariant second moment code format". Journal of the Engineering Mechanics Division, 100(1), pp. 111-121. 1974.

[11] Liu, P.-L. and Der Kiureghian, A. "Optimization algorithms for structural reliability". Structural Safety, 9(3), pp. 161-177. 1991. https:/doi. org/10.1016/0167-4730(91)90041-7

[12] Rackwitz, R., Flessler, B. "Structural reliability under combined random load sequences". Computers \& Structures, 9(5), pp. 489-494. 1978. https://doi.org/10.1016/0045-7949(78)90046-9

[13] Zhang, Y., Der Kiureghian, A. "Two improved algorithms for reliability analysis". In: Reliability and Optimization of Structural Systems, Springer, Boston, pp. 297-304. 1995. https://doi.org/10.1007/978-0-38734866-7_32

[14] Gandomi, A. H., Yang, X.-S., Talatahari, S., Alavi, A. H. "Metaheuristic applications in structures and infrastructures". Elsevier. 2013. https:// doi.org/10.1016/c2011-0-08778-1

[15] Wang, J., Ghosn, M. "Linkage-shredding genetic algorithm for reliability assessment of structural systems". Structural Safety, 27(1), pp. 49-72. 2005. https://doi.org/10.1016/j.strusafe.2004.06.001

[16] Yan, H., Guang-Wei, M., Zhen-Ping, Z., Feng, L. "Structural reliability analysis based on imperialist competitive algorithm". In: Fourth International Conference on Intelligent Systems Design and Engineering Applications, pp. 570-574, 2013. https://doi.org/10.1109/isdea.2013.535

[17] Zhao, H., Ru, Z., Chang, X., Li, S. "Reliability analysis using chaotic particle swarm optimization" Quality and Reliability Engineering International, 31(8), pp.1537-1552. 2015. https://doi.org/10.1002/qre.1689

[18] Kaveh, A., Ilchi Ghazaan, M. "Structural reliability assessment utilizing four metaheuristic algorithms". Iran University of Science \& Technology, 5(2).pp. 205-225, 2015. http://ijoce.iust.ac.ir/article-1-210-en.pdf

[19] Cornell, C. A. "A probability based structural code". ACI-Journal, 66(12), pp. 974-985. 1969. https://doi.org/10.14359/7446

[20] Basler, E. "Untersuchungen über den Sicherheitsbegriff von Buawerken", "Investigations on the security concept of buildings". Doctoral thesis, ETH Zürich, Switzerland, 1961. (in German). https://doi. org/10.3929/ethz-a-000099666

[21] Rosenblatt, M. "Remarks on a multivariate transformation". The Annals of Mathematical Statistics, 23(3), pp. 470-472. 1952. https://doi. org/10.1214/aoms/1177729394

[22] Jia, G., Wang, Y., Cai, Z. and Jin, Y. "An improved $(\mu+\lambda)$-constrained differential evolution for constrained optimization". Information Sciences, 22, pp. 302-322, 2013. https://doi.org/10.1016/j.ins.2012.01.017

[23] Coello Coello, C. A. "Theoretical and numerical constraint-handling techniques used with evolutionary algorithms: a survey of the state of the art". Computer Methods in Applied Mechanics and Engineering, 191(11-12), pp. 1245-1287. 2002. https://doi.org/10.1016/s0045-7825(01)00323-1
[24] Deb, K. "An efficient constraint handling method for genetic algorithms". Computer Methods in Applied Mechanics and Engineering, 186(2-4), pp. 311-338. 2000. https://doi.org/10.1016/s0045-7825(99)00389-8

[25] Ho-Huu, V., Nguyen-Thoi, T., Nguyen-Thoi, M. H., Le-Anh, L. "An improved constrained differential evolution using discrete variables (D-ICDE) for layout optimization of truss structures". Expert Systems with Applications, 42(20), pp. 7057-7069. 2015. https://doi.org/10.1016/j. eswa.2015.04.072

[26] Ho-Huu, V., Nguyen-Thoi, T., Le-Anh, L., Nguyen-Trang, T. "An effective reliability-based improved constrained differential evolution for reliability-based design optimization of truss structures". Advances in Engineering Software, 92, pp. 48-56, 2016. https://doi.org/10.1016/j. advengsoft.2015.11.001

[27] Storn, R., Price, K. "Differential evolution - a simple and efficient heuristic for global optimization over continuous spaces". Journal of Global Optimization, 11(4), pp. 341-359. 1997. https://doi. org/10.1023/A:1008202821328

[28] Kukkonen, S., Lampinen, J. "Constrained real-parameter optimization with generalized differential evolution". IEEE International Conference on Evolutionary Computation, Vancouver, BC, 2006, pp. 207-214. 2006. https://doi.org/10.1109/cec.2006.1688310

[29] Grooteman, F. "Adaptive radial-based importance sampling method for structural reliability". Structural Safety, 30(6), pp. 533-542. 2008. https://doi.org/10.1016/j.strusafe.2007.10.002

[30] Engelund, S. and Rackwitz, R. "A benchmark study on importance sampling techniques in structural reliability". Structural Safety, 12(4), pp. 255-276. 1993. https://doi.org/10.1016/0167-4730(93)90056-7

[31] Rajashekhar, M. R., Ellingwood, B. R. "A new look at the response surface approach for reliability analysis". Structural Safety, 12(3), pp. 205-220. 1993. https://doi.org/10.1016/0167-4730(93)90003-j

[32] Weingarten, V., Seide, P. "Buckling of thin-walled truncated cones". NASA Space Vehicle Criteria (Structures), NASA SP-8019, Washington, DC., 1968. https://ntrs.nasa.gov/archive/nasa/casi.ntrs.nasa. gov/19690014753.pdf

[33] Rashki, M., Miri, M., AzhdaryMoghaddam, M. "A new efficient simulation method to approximate the probability of failure and most probable point". Structural Safety, 39, pp. 22-29. 2012. https://doi.org/10.1016/j. strusafe.2012.06.003

[34] Ranganathan, R. "Reliability analysis and design of structures". Tata McGraw-Hill Publishing Company Limited, New Delhi, 1990.

[35] Lan, C.-m., Li, H., Peng, J.-y., Sun, D.-b."A structural reliability-based sensitivity analysis method using particles swarm optimization: relative convergence rate". Journal of Zhejiang University-SCIENCE A, 17(12), pp. 961-973. 2016. https://doi.org/10.1631/jzus.a1500255

[36] Devictor, N. "Fiabilitéet mécanique: méthodes FORM/SORM et couplages avec des codes d'éléments finis par des surfaces de réponseadaptatives". University of Clermont-Ferrand 2, France. 1996.

[37] Nguyen, X. S., Sellier, A., Duprat, F., Pons, G. "Adaptive response surface method based on a double weighted regression technique". Probabilistic Engineering Mechanics, 24(2), pp. 135-143. 2009. https://doi. org/10.1016/j.probengmech.2008.04.001 